SERIE B - INFORMATIK

\title{
On the Number of Simple Cycles in Planar Graphs
}

\author{
Helmut Alt* \\ Ulrich Fuchs* \\ Klaus Kriegel* \\ B 97-08 \\ September 1997
}

\begin{abstract}
Let $C(G)$ denote the number of simple cycles of a graph $G$ and let $C(n)$ be the maximum of $C(G)$ over all planar graphs with $n$ nodes. We present a lower bound on $C(n)$ constructing graphs with at least $2.28^{n}$ cycles. Applying some probabilistic arguments we prove an upper bound of $3.37^{n}$.

We also discuss this question restricted to the subclasses of grid graphs, bipartite graphs, and of 3-colorable triangulated graphs.
\end{abstract}




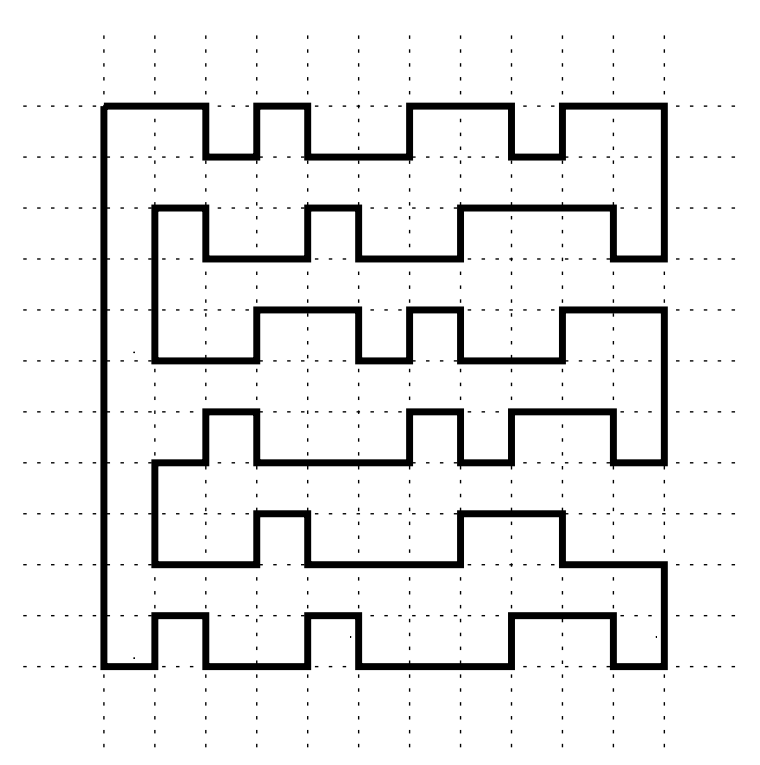

Figure 1: Blum and Hewitt's construction

\section{The Problem}

The question addressed in this note came up in connection with problems in the theory of VLSI-layout and the computation of rectilinear Steiner trees [4], but is also a very natural one in graph theory:

\section{How many different simple cycles can there be in a planar graph with $n$ nodes?}

Let us call this number $C(n)$. A very simple upper bound can be obtained by the following observation: By Eulers formula, a planar embedding of a graph with $n$ nodes can have at most $2 n-4$ faces. Any simple cycle encloses a subset of these faces and is identified by this subset. Consequently,

$$
C(n) \leq 2^{2 n-4}=\frac{1}{16} \cdot 4^{n}
$$

Observe that this upper bound was established making use of the dual version of our problem: How many simply connected regions can be obtained by unifying countries in a planar map? A special case of this question was studied by Blum and Hewitt [1]. Their aim was to estimate the number of simply connected patterns in an $k \times k$-grid. Thus, the construction in [1] proved a first lower bound on $C(n)$.

In particular the following set of simple cycles is constructed (see Figure 1): The leftmost column of the $k \times k$-grid belongs to every cycle, then in rows 1 and 2 we walk to the right and for each step rightwards we have the choice between row 1 and 2 . We insert vertical steps between the horizontal ones, if necessary. In the same way we use rows 3 and 4 to walk back to the left. This procedure continues until, in a snakelike fashion, we have traversed the whole grid and are back at the lower end of the leftmost column. For any two rows, we have $k-2$ choices, so altogether there are $2^{k / 2 \cdot(k-2)}$ different cycles of 

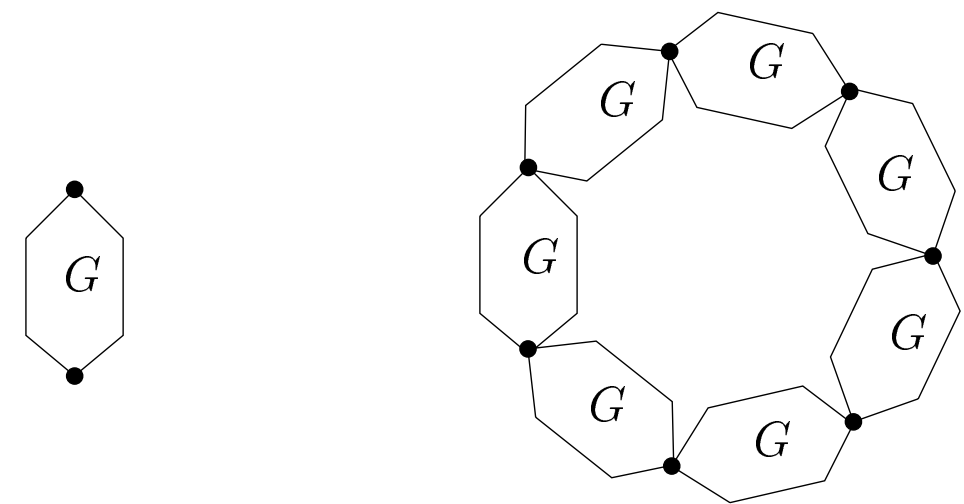

Figure 2: The Graph $G_{t}$

this kind. Consequently, since the graph has $n=k^{2}$ nodes,

$$
C(n) \geq \sqrt{2}^{n-2 \sqrt{n}}
$$

for infinitely many $n \in \mathbb{N}$.

This lower bound can be improved in an obvious way: Triangulating all tiles of the grid by a diagonal one has three choices for any step: row 1, row 2 or the diagonal. Thus we get

$$
C(n) \geq \sqrt{3}^{n-2 \sqrt{n}}
$$

for infinitely many $n \in \mathbb{N}$.

So $C(n)$ is exponential in $n$ and by (1) and (2) the basis of the exponent lies somewhere between $\sqrt{3}$ and 4 . In this paper we will close this gap to some extend but not completely. In a recent paper Ding [2] gave a minor based characterization of graph classes, such that the number of cycles is polynominal. In fact, one can observe that our exponetial lower bound examples correspond to one of the forbidden minor types in a very natural way.

\section{Lower Bounds}

In order to get a better lower bound on $C(n)$ we first show the following lemma:

Lemma 1 Let $G$ be a planar graph with $k$ nodes and $a, b$ be two nodes of $G$ incident to the outer face for some planar embedding of $G$. Let $s$ be the number of simple paths between $a$ and $b$. Then

$$
C(n) \geq(\sqrt[k-1]{s})^{n}
$$

for infinitely many $n \in \mathbb{N}$.

Proof: Consider the planar embedding where $a, b$ are incident to the outer face. Then for any $t \in \mathbb{N}, t \geq 3$ we can construct a graph $G_{t}$ with $n=t(k-1)$ nodes, as shown in Figure 2 . 


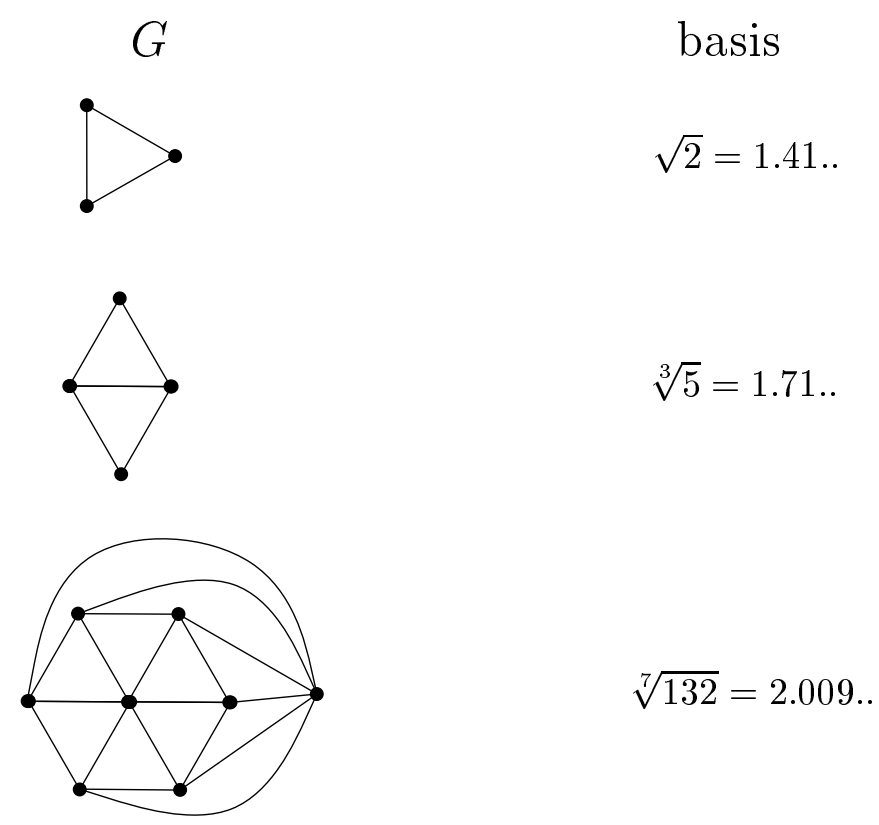

Figure 3: Lower bounds obtained by simple graphs

Since there are $s$ simple paths from $a$ to $b$, there are at least $s^{t}$ simple cycles within $G_{t}$. Consequently,

$$
C(n) \geq s^{t}=s^{n /(k-1)}=(\sqrt[k-1]{s})^{n}
$$

Using Lemma 1 we obtain nontrivial lower bounds already for very simple graphs $G$, in fact the previous bound $\sqrt{2}$ is achieved already when $G$ is a triangle, see Figure 3. The best lower bound on $C(n)$ we have, is obtained by applying Lemma 1 to the graph shown in Figure 4. Observe, that it is sufficient for $a$ and $b$ to be incident to some face, since it can be turned into the outer face by a homeomorphism. It also makes sense to triangulate the graph, since adding edges can only increase the lower bound. So without loss of generality $a, b$ are the endpoints of an edge. For the graph $G$ of Figure 4 we determined the number of simple paths between endpoints of edges by computer and found the maximum $s=10,722,120,801$ between the vertices $a, b$ indicated in the picture. For the basis of the exponent we obtain $\sqrt[28]{s}=2.281 \ldots$, so we have as a lower bound for $C(n)$ :

Theorem $1 C(n) \geq \alpha^{n}$, where $\alpha=\sqrt[28]{10,722,120,801}=2.281 \ldots$ for infinitely many $n \in \mathbb{N}$.

Our computer experiments indicated that the value of $\alpha$ can probably be increased by considering larger and larger graphs of the form of the graph $G$ in Figure 4. We stopped with $G$, because of the excessive computation time. For $G$ we needed 30 hours on a SPARC-Station-10/512 computer to determine $s$.

The method to obtain non-trivial upper bounds, that will be presented in the next section, gives better results for 3-colorable graphs. Hence we also had a look on the lower 


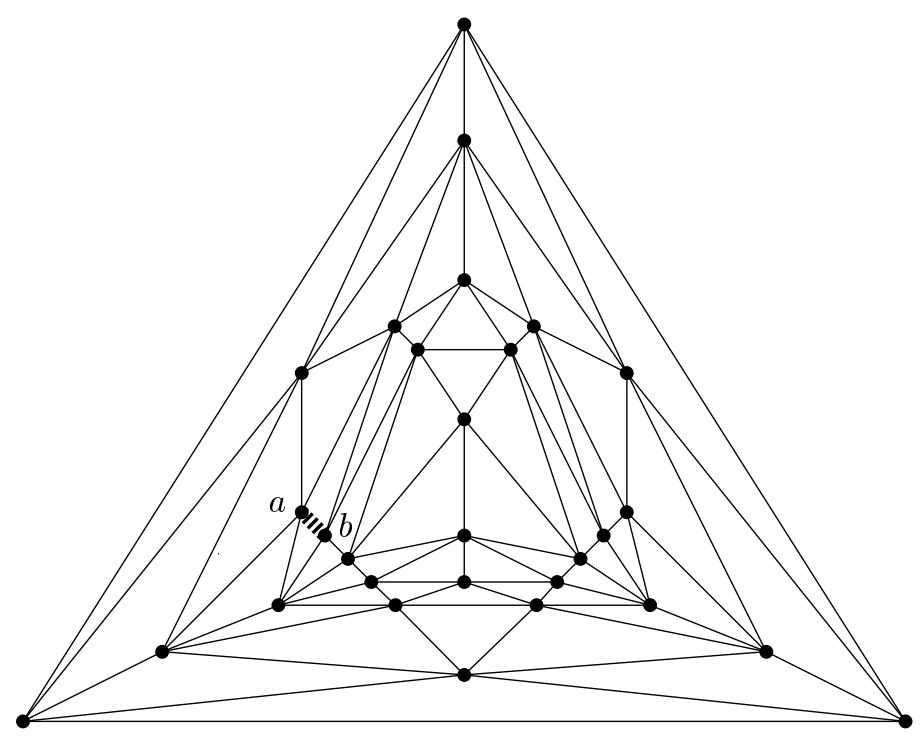

Figure 4: Graph $G$ yielding a bound of $2.275 \ldots$

bounds for this class of graphs. A triangulated graph is 3-colorable iff the degree of each node is even. This was already mentioned in [5], proofs and more details can be found in [6] and [7]. We counted (36 hours of computation time) in a graph with 29 nodes, all of degree four or six, 8,133,124,602 paths between two adjacent vertices. Observe, that if $G$ has only nodes of even degree and if $t$ is three times a power of 2 the Graph $G_{t}$ can be triangulated such that all nodes have even degree. Evaluating $\sqrt[28]{8,133,124,602}$ we get:

Theorem 2 For infinitely many natural numbers $n$ there is a 3-colorable planar graph with $\Omega\left(2.259^{n}\right)$ simple cycles.

We also tested some bipartite i.e. 2-colorable graphs. For a graph with 36 nodes (more or less a $6 \times 6$-grid) we found $38,855,881$ paths between two adjacent ones. For even $t G_{t}$ remains bipartite and hence we get:

Theorem 3 For infinitely many natural numbers $n$ there is a bipartite planar graph with $\Omega\left(1.647^{n}\right)$ simple cycles.

\section{Upper bounds}

In order to get a better upper bound than $O\left(4^{n}\right)$ for $C(n)$ we use a probabilistic argument. Suppose we have some planar embedding for a graph $G=(V, E)$ and we color the faces randomly under uniform distribution with 2 colors ('black' and 'white'). If we are 'lucky' the total black area is simply connected, i.e. it has no holes and its interior is connected. Then the boundary of this area is a simple cycle (see Figure 5). Let us call such a coloring 'valid'. We will determine an upper bound on the probability of this event. Let us again assume that $G$ is triangulated, clearly any upper bound on $C(n)$ under this assumption holds for general planar graphs as well. Consider some node $v$ of $G$ and inspect its incident faces in clockwise order (see Figure 5). Assume that there are both, black and white faces incident to $v$. In the 'valid' case it is necessary that the black faces start at some edge $e_{1}$, 


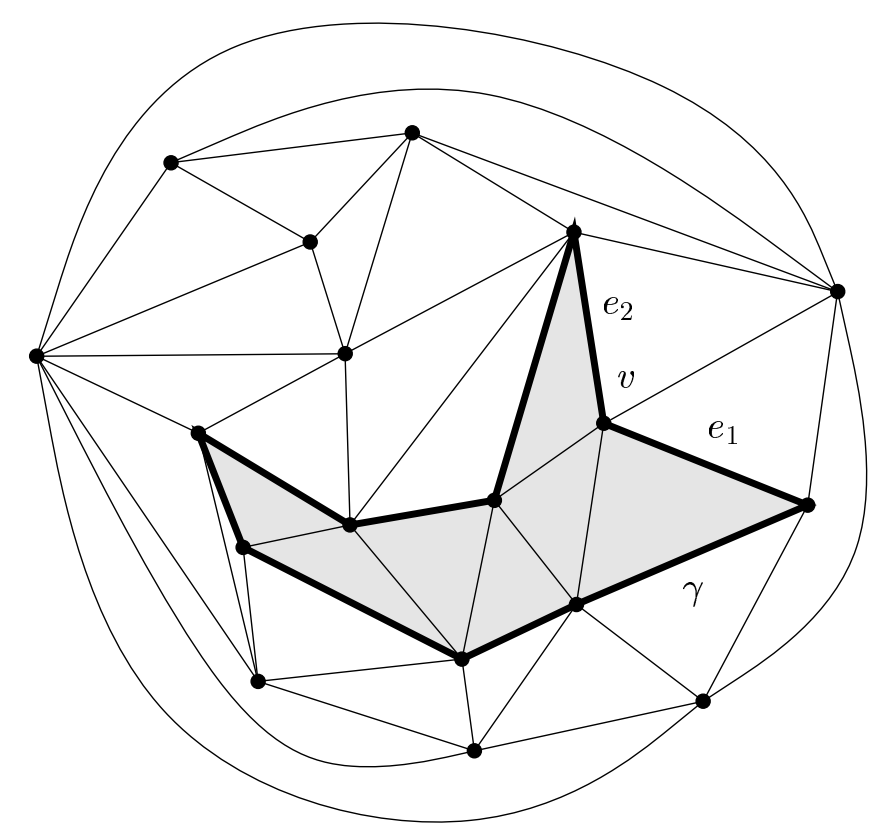

Figure 5: The total black area is simply connected and identifies a simple cycle $\gamma$

form a connected sequence and end at some edge $e_{2}$. Let us call $v$ 'good' if this is the case or if all faces incident to $v$ have the same color. The number of possible colorings of the incident faces making some node $v$ of degree $d$ good is

$$
2+d(d-1)
$$

namely, coloring all faces black or all white or selecting edges $e_{1}$ and $e_{2}$ as described before and coloring black exactly the faces between $e_{1}$ and $e_{2}$. Therefore, the probability that $v$ is good is

$$
\frac{d(d-1)+2}{2^{d}}
$$

An upper bound for the probability that a coloring is valid, is the probability $p_{\text {good }}$ that all nodes are good, since the latter condition is necessary for the former. If the goodness of all nodes were independent events we would obtain this probability by the product

$$
\prod_{v \in V} \frac{d(v)(d(v)-1)+2}{2^{d(v)}}
$$

where $d(v)$ is the degree of a node $v$. The denominator of this product equals

$$
2^{\sum_{v \in V} d(v)}=2^{6 n-12},
$$

since $\sum_{v \in v} d(v)$ equals twice the number of edges. The enumerator in (3) is maximal under the constraint $\sum_{v \in V} d(v)=6 n-12$ if all $d(v)$ are equal. To see this, consider the 
function $f(d)=d(d-1)+2$. Straightforward calculations yield for $0<\varepsilon<d$

$$
\begin{aligned}
f(d+\varepsilon) f(d-\varepsilon) & =(f(d))^{2}+\varepsilon^{2}\left(\varepsilon^{2}+3-2 d^{2}+2 d\right) \\
& <(f(d))^{2}+\varepsilon^{2}\left(-d^{2}+2 d-1+4\right) \\
& =(f(d))^{2}+\varepsilon^{2}\left(4-(d-1)^{2}\right) \\
& \leq(f(d))^{2}
\end{aligned}
$$

since $G$ is triangulated and therefore $d \geq 3$. So, whenever two $d(v)$ in (3) differ by some amount $2 \varepsilon$ we can increase the value by increasing one by $\varepsilon$ and decreasing the other by $\varepsilon$, thus, making them equal. Consequently, the expression in (3) is maximized by letting $d(v)=\frac{6 n-12}{n} \leq 6$, so we have

$$
\prod_{v \in V} \frac{d(v)(d(v)-1)+2}{2^{d(v)}} \leq 2^{12}\left(\frac{6 \cdot 5+2}{2^{6}}\right)^{n}=2^{12}\left(\frac{1}{2}\right)^{n} .
$$

However, this is not an upper bound on the probability of obtaining a valid coloring, since the events of the nodes being good are not independent. Let $A_{u}$ denote the event that the vertex $v$ is good for a random coloring of the faces, then it is not hard to check that the events $A_{u}$ and $A_{v}$ are not independent for adjacent vertices $u$ and $v$. However, if we choose an independent set of vertices $V$, then the events $A_{v}(v \in V)$ are mutually independent because the sets of faces surrounding the vertices of $V$ are disjoint. Note that this is only true for triangulated planar graphs. By the four-color-theorem the nodes of $G$ can be partitioned into 4 independent sets $V_{1}, \ldots, V_{4}$. Let $V_{i}$ be that one which minimizes

$$
\prod_{v \in V_{i}} \frac{d(v)(d(v)-1)+2}{2^{d(v)}} .
$$

So by (4) we have

$$
p_{\text {good }} \leq \prod_{v \in V_{i}} \frac{d(v)(d(v)-1)+2}{2^{d(v)}} \leq \sqrt[4]{2^{12}\left(\frac{1}{2}\right)^{n}}=8\left(\sqrt[4]{\frac{1}{2}}\right)^{n}
$$

Since there are $2 n-4$ faces, there are $2^{2 n-4}$ colorings of the faces. This implies the following upper bound on the number of cycles.

\section{Theorem 4}

$$
C(n) \leq p_{\text {good }} \cdot \frac{1}{16} \cdot 4^{n} \leq \frac{1}{2}(\sqrt[4]{1 / 2} \cdot 4)^{n}
$$

and thus the basis is at most $\sqrt[4]{1 / 2} \cdot 4=3.363 \ldots$

The general upper bound can be improved for 3-colorable triangulated planar graphs in an obvious way. Since there are only three color classes we will find one such that

$$
\prod_{v \in V_{i}} \frac{d(v)(d(v)-1)+2}{2^{d(v)}} \leq{\sqrt[3]{2^{12}\left(\frac{1}{2}\right)^{n}}}^{n}=16 \cdot \sqrt[3]{1 / 2}^{n} .
$$


Corollary 1 The number of simple cycles in a 3-colorable triangulated planar graph is bounded by

$$
16 \sqrt[3]{1 / 2}^{n} \cdot 2^{2 n-4}=(\sqrt[3]{1 / 2} \cdot 4)^{n} \leq 3.175^{n}
$$

Now one could expect a further improvement of the upper bound for 2-colorable (i. e. bipartite) graphs. In fact we will obtain a much better bound, but we cannot argue as before, for the following reason: A bipartite graph is not triangulated and therefore the events $A_{v}$ are no longer independent for vertices of the same color. On the other hand bipartite graphs have at most $n-2$ faces and thus the number of cycles can be at most $2^{n-2}$. Hence, our aim is to improve this bound by probabilistic arguments.

Analogously to the general case we may assume that all faces of the graph $G$ are cycles of length four. Then by Euler's formula we have $n$ vertices, $2 n-4$ edges and $n-2$ faces. Again, let $A_{v}$ denote the event that a random coloring of the faces is good at $v$. Multiplying the probabilities of all events $A_{v}$ we get

$$
\prod_{v \in V} \frac{2+d(v)(d(v)-1)}{2^{d(v)}} \leq \frac{2+4 \cdot 3}{2^{4 n-8}}=2^{8} \cdot\left(\frac{7}{8}\right)^{n} .
$$

Now we want to partition $V$ in such a way that each corresponding subproduct represents mutually independent events $A_{v}$. Let $V=V_{a} \cup V_{b}$ be the partition of $V$ given by the bipartiteness of $G$. We define auxiliary graphs $G_{a}\left(G_{b}\right)$ with vertex sets $V_{a}\left(V_{b}\right)$ and edge sets $E_{a}\left(E_{b}\right)$ consisting of all face diagonals between vertices from $V_{a}\left(V_{b}\right)$. Both graphs are planar. So we can color $V_{a}$ with the colors $\{1,2,3,4\}$ and $V_{b}$ with the colors $\{5,6,7$, $8\}$. It follows that for two vertices $v, u$ of the same color the sets of the faces surrounding them are disjoint and thus the events $A_{v}$ and $A_{u}$ are independent. Consequently, there is a color class $V_{i}$ such that

$$
\prod_{v \in V} \frac{2+d(v)(d(v)-1)}{2^{d(v)}} \leq{\sqrt[8]{2^{8} \cdot\left(\frac{7}{8}\right)}}^{n}=2 \cdot \sqrt[8]{\frac{7}{8}}^{n}
$$

Taking into account that there are $2^{n-2}$ face colorings we get the following

Theorem 5 The number of simple cycles in a bipartite planar graph is bounded by

$$
2 \cdot \sqrt[8]{\frac{7}{8}}^{n} \cdot 2^{n-2} \leq\left(\sqrt[8]{\frac{7}{8}} \cdot 2\right)^{n} \leq 1.967^{n} .
$$

For grid graphs one can get a slightly better bound because in this case $G_{a}$ and $G_{b}$ are bipartite, which implies a partition of $V$ into four color classes.

Corollary 2 The number of cycles in an $m \times k-\operatorname{grid}(n=m \cdot k)$ is bounded by

$$
4 \cdot\left(\sqrt[4]{\frac{7}{8}}\right)^{n} \cdot 2^{n-(m+k-1)}<\sqrt[4]{14}^{n} \leq 1.935^{n} .
$$




\section{Conclusions}

The following table summarizes the lower and upper bounds on the maximal number $C(n)$ of cycles in planar graphs on $n$ vertices:

\begin{tabular}{|c|c|c|}
\hline graph class & lower bound & upper bound \\
\hline \hline general planar & $2.281^{n}$ & $3.364^{n}$ \\
\hline 3-colorable, triangulated & $2.259^{n}$ & $3.175^{n}$ \\
\hline bipartite & $1.647^{n}$ & $1.967^{n}$ \\
\hline grid graphs & $1.414^{n}$ & $1.935^{n}$ \\
\hline
\end{tabular}

The remaining challenge in this context is of course to further close the gaps between the lower and upper bounds. As already mentioned one can obtain minor improvements of the lower bounds counting the number of $a b$-paths for larger graphs. A similar lower bound of $2.279^{n}$ has been proved recently by Chrobak and Eppstein [3]. Their method does not require an excessive path counting but instead they have to compute the eigenvalues of a $6 \times 6$-matrix.

An other way to get minor improvements of the lower bounds consists in triangulating the inner and the outer face of the graph $G_{t}$ constructed in the proof of Lemma 1. However, we could not improve the basis of the lower bound much more than by the factor of 1.001 .

It would be interesting to prove an upper bound for not necessarily triangulated 3colorable graphs which is better than the general one. Note that the additional assumption that the graphs have to be triangulated was necessary to get 3 sets of mutually independent events. On the other hand there are 3-colorable graphs for which no 3-colorable triangulations exist.

There is a natural approach to improve the upper bounds. In fact our previous upper bounds estimate the number of face colorings which are good in every vertex. In the dual version this means that we have bounds on the number of families of disjoint cycles in a graph. So one could try to estimate the probability that a random family of disjoint cycles in a graph consists of exactly one cycle. Consequently one improves the previous upper bound by multiplying it with the probability. So far we did not succeed in this way.

Finally we remark that it could be helpful to have an upper bound on the number of Hamiltonian cycles in planar graphs. Indeed, a bound of the form $\alpha^{n}$ would imply $C(n) \leq(\alpha+1)^{n}$ applying the bound to each induced subgraph with $k$ vertices $k=1, \ldots, n$ and making use of the binomial formula.

\section{Acknowledgement}

We would like to thank Frank Hoffmann for several valuable hints concerning this research.

\section{References}

[1] M. Blum, C. Hewitt, Automata on a 2-dimensional tape, 8th IEEE Conf. on SWAT, 1967, 155-160.

[2] G. Ding, Bounding the Number of Circuits of a Graph, Combinatorica 16(3), 1996, 331-341. 
[3] D. Eppstein, Pesonal Communications, 1997

[4] U. Fössmeier, M. Kaufmann, On Exact Solutions for the Rectilinear Steiner Problem, Part I: Theoretical Results, Technical Report, Computer Science Institute, Universität Tübingen, 1996.

[5] P. J. Heawood, Map Color Theorem, Quarterly Journal of Pure and Applied Mathematics 24, 1890, 332-338.

[6] T. R. Jensen, B. Toft, Graph Coloring Problems, Wiley, New York, 1995.

[7] R. Steinberg, The State of the Three Color Problem, Annals of Discrete Mathematics 55, 1993, 211-248. 